\title{
Monte Carlo Simulation for the Majorana Neutrinoless Double-beta Decay Experiment
}

\author{
R. Henninga for the Majorana Collaboration \\ ${ }^{\text {a} L a w r e n c e ~ B e r k e l e y ~ N a t i o n a l ~ L a b o r a t o r y, ~} 1$ Cyclotron Road, Berkeley, CA, USA
}

The Majorana experiment is a proposed HPGe detector array that will primarily search for neutrinoless doublebeta decay and dark matter. It will rely on pulse-shape discrimination and crystal segmentation to suppress backgrounds following careful materials selection. A critical aspect of the design phase of Majorana is a reliable simulation of the detector response, pulse formation, and its radioactive backgrounds. We are developing an adaptable and complete simulation based on GEANT 4 to address these requirements and the requirements of a modern, large collaboration experiment. The salient aspects of the simulation are presented. The Majorana experiment is presented in a parallel poster by Kareem Kazkaz.

The Majorana experiment is a proposed $500-\mathrm{kg}$ enriched, segmented, HPGe detector array that will primarily search for neutrinoless double-beta decay and dark matter. It will rely on pulse-shape discrimination and crystal segmentation to suppress backgrounds following careful materials selection. The Majorana experiment is described in a parallel poster by Kareem Kazkaz.

Several physical processes and detector geometries have to be simulated for the R\&D and operational phases of Majorana. These include energy deposition from radioactive background sources, cosmic rays, and signal sources; for which low-energy electromagnetic and neutron interaction processes are especially important. Pulseshape formation in crystals has to be simulated to help optimize the crystal segmentation scheme and geometry. Different active and passive shielding configurations have to studied and optimized. Generators for the radioactive decay backgrounds, as well as signals, have to be developed or borrowed. Activation in detector materials and crystals have to be simulated to understand induced background from cosmic ray exposure at the surface. Finally, different crystal packing arrangements have to be studied for optimal gamma and neutron veto capabilities.

The simulation package must also fulfill the requirements of large collaboration and persist for the lifetime of the experiment, hence it must be well-documented, planned and maintained. The complexity of this software task requires the use of professional programming techniques and tools. These goals are realized through the integration of several packages and tools into a unified, $\mathrm{C}++$-based framework. Many different detector geometries and simulation studies will be performed, making full use of the powerful object-oriented and abstraction capabilities of $\mathrm{C}++$. Particle tracking, geometries and most physical process are simulated using Geant 4 [1]. Pulse-shapes are simulated with custom software that requires an electric field solver, for which we have selected Maxwell3D. The simulation package's reference documentation is generated by DOxygen[2], while the User's Guide is written in Docbooks[3]. The database is based on opensource PostgreSQL[4] and contains detector description data.

The simulation is under development, and initial physics results are currently being utilized by the Majorana collaboration for R\&D.

\section{REFERENCES}

1. S. Agostinelli et al NIM A 506 (2003) 250.

2. http://www.doxygen.org

3. http://www.oreilly.com/catalog/docbook

4. http://www.postgresql.org 